\title{
NON-ROBUSTNESS WITH RESPECT TO INTERVENTION COSTS IN OPTIMAL CONTROL*
}

\author{
Nils Chr. Framstad ${ }^{\dagger}$
}

\begin{abstract}
It is well known that a wide range of impulse control problems are continuous, but not differentiable, with respect to intervention cost as it approaches zero. We show a similar non- $\mathrm{C}^{1}$ result, assuming that the problem and the value function are sufficiently nice. The result is that if the value function is locally $\mathrm{C}^{1}$ in the state variable, then it does not admit first order approximation in the intervention cost. Our analysis also covers problems with reflection (i.e. vanishing impulse).
\end{abstract}

\section{Motivation}

This paper concerns the non-regularity of the value or cost function associated to impulse control problems, with respect to intervention costs. Optimal stochastic control problems where the optimal strategy is a reflection is covered as well, whether they are stochastic and singular or deterministic and absolutely continuous.

The introduction of intervention costs to a problem will typically make it more difficult to solve the problem explicitly. If the intervention cost $\kappa$ is small, one may want to take the known solution to the case $\kappa=0$ as an approximation. [1] shows that such a perturbation in $\kappa$ is continuous at $\kappa=0$ for a class of problems driven by an Itô diffusion. It turns out, however, that frequently there is no first order approximation and the $\kappa$-derivative may explode at zero. This non-robustness property is treated in [2] and [3]. Their approach is to formulate a class of (jump-) diffusion models and deduce the property from the HamiltonJacobi-Bellman sufficient conditions for optimality. The approach presented herein is different; it assumes ad hoc some structure on the optimal value function, namely differentiability within the interior of the continuation region and that as the cost parameter vanishes, so does optimal intervention magnitude («per intervention» that is; typically, one will then intervene more frequently.) The drawback is that this is not à priori known, although a wide class of problems do satisfy the «high contact» principle, namely differentiability even across the boundaries of the continuation region (see remark at the end of the paper.) On the other hand, it is reasonable to claim that if the known limiting solution at $\kappa=0$ is differentiable (wrt. the state variable) while the solution for $\kappa \neq 0$ is not, or if the optimal strategy is not in the appropriate sense continuous at $\kappa=0$, then approximating the latter problem from the former by linearization is unsatisfactory. Within the range of problems covered by our analysis, the result supports the view that as such an approximation is «bad,» that the presence of real-life intervention costs should be modeled even if they are small, and that the theory of impulse control should be considered indispensable even for small intervention costs.

\footnotetext{
*Supported by the Research Council of Norway.

${ }^{\dagger}$ Dept. of Mathematics, University of Oslo, Box 1053 Blindern, N-0316 Oslo, Norway. ncf@math.uio.no
} 


\section{The setup and the result:}

We assume that we have a state variable $x$ and a regime variable $\chi$. The «regime» is thought of as a variable which may be switched at will. We assume two regimes, not necessarily distinct, so $\chi \in\{0,1\}$, and merely note that the generalization to more than two is straightforward.

The «state,» on the other hand, has a dynamics of its own, e.g. as a Markov process; nevertheless, we may intervene in the state as well, shifting it to an new state $\xi$, each of whose components may be arbitrary or not affected at all, i.e.

$$
\text { if } x=(y, z) \text {, then } \xi=(y, \zeta) \text { where we are free to choose } \zeta \text {. }
$$

We shall assume that there are «costs» involved in changing the state; in a simple setting, we may think of the cost associated with changing from state $x$, regime $\chi$ to state $\xi$, regime $1-\chi$ as $k_{\chi}(x, \xi) \cdot \kappa$ where the nonnegative parameter $\kappa$ will be the object of our analysis; we shall consider what happens when $\kappa \searrow 0$. However, we will as a generalization allow the intervention cost to have a higher order term $K$, so that the cost of the above intervention will be

$$
K_{\chi}(\kappa, x, \xi)+k_{\chi}(x, \xi) \cdot \kappa \quad \text { where } \frac{\partial K_{\chi}}{\partial \kappa}\left(0^{+}, x, \xi\right)=0 .
$$

We shall stick to the terminology «costs» even though it may have either sign. We shall however need to rule out switching back and forth instantaneously for $\kappa \neq 0$. More precisely, we shall assume that the continuation regions $D_{\chi}=D_{\chi, k}$, defined to be the set of $x$ such that once in state $\chi$ it is not optimal to intervene, satisfies the following regularity assumption:

\section{ASSUMPTION}

For any $\epsilon>0$ and both $\chi$, then for all $\kappa$ small enough there are open sets $O_{\chi, \kappa} \subseteq D_{\chi, \kappa}^{\mathrm{c}}$ within distance $d\left(\bar{x}, O_{\chi, \kappa}\right)<\epsilon$. (Here, ${ }^{\mathrm{c}}$ denotes complement.)

Furthermore, at the point $\bar{x}$ we require

$$
k_{0}(\bar{x}, \bar{x})+k_{1}(\bar{x}, \bar{x}) \neq 0
$$

i.e., if we disregard the higher order terms $K$, there will still be a proper loss associated to switching back and forth at the same time.

Our setup models the following situation: for $\kappa=0$, we have a usual continuous control problem; at the point $\bar{x}$, one will apply some (gratis) control. As the control is associated to a cost, introduced as a nonlinear function of the parameter $\kappa$ but with at most linear growth at $\kappa=0$, it is no longer optimal to continuously exercise control at this point, but to wait until $x$ exits the continuation region $D_{\chi}$ associated to the current regime $\chi$.

We assume that the control problem has value function $\phi$, which we take to be a function $\phi(\kappa, x, \chi)$ of $\kappa$ as well. For all $x_{\chi} \notin D_{\chi}$, it is optimal to switch from regime $\chi$ to $1-\chi$, and to some new state $\xi$. Thus,

$$
\phi\left(k, x_{\chi}, \chi\right)=\sup _{\zeta}\left\{\phi(\kappa, \xi, 1-\chi)-K_{\chi}\left(\kappa, x_{\chi}, \xi\right)-k_{\chi}\left(x_{\chi}, \xi\right) \cdot \kappa\right\} .
$$


By (1), then under the appropriate differentiability conditions we have the first order condition

$$
\nabla_{\zeta} \phi\left(\kappa, y, \zeta_{\chi}(\kappa, x), 1-\chi\right)-\nabla_{\zeta} K_{\chi}\left(\kappa, x_{\chi}, y, \zeta_{\chi}(\kappa, x)\right)-\nabla_{\zeta} k_{\chi}\left(x_{\chi}, y, \zeta_{\chi}(\kappa, x)\right) \cdot \kappa=0
$$

for the optimal $\xi=\xi_{\chi}\left(\kappa, x_{\chi}\right)=\left(y, \zeta_{\chi}\left(\kappa, x_{\chi}\right)\right.$. The following assumption is almost trivial:

$$
\xi_{\chi}\left(\kappa, x_{\chi}\right) \in D_{1-\chi, k} \forall\left(\kappa, x_{\chi}\right)
$$

(if not, rewrite the problem.) The next, however, is crucial:

$$
d\left(O_{\chi, \kappa}, \xi_{\chi}\left(\kappa, x_{\kappa}\right)\right) \underset{\kappa \searrow 0}{\longrightarrow} 0 \text { for } x_{\kappa} \in O_{\chi, \kappa} \text { arbitrary }
$$

for the sets $O$ in the Assumption. This condition is a bit restrictive as it ad hoc assumes that interventions get small as the costs do, but covers a wide class of problems. In the spirit of this paper, one may maintain that if (6) does not hold, then the zero cost problem yields a bad approximation, as the optimal strategy is (in a heuristic sense) non-robust with respect to intervention costs.

Given the known connections between optimal stopping and singular optimal stochastic control, see [4] and the numerous references therein, it is not surprising that the result we are about to present covers some singular stochastic control problems as well. The setup has so far been of impulse control. However, an optimal control problem (deterministic or stochastic) with reflection may (or may not) be obtained if the regimes are not distinct, depending on the cost functions $K$ and $k$. Therefore the following theorem also covers a wide range of control problems with reflection, with $\phi$ independent of $\chi$ :

\section{THEOREM}

Suppose the Assumption and (1) - (6) hold, that $\xi \mapsto \phi(\kappa, \xi, 1-\chi)-K_{\chi}\left(\kappa, x_{\chi}, \xi\right)-k_{\chi}\left(x_{\chi}, \xi\right) \cdot \kappa$ is differentiable on the interior of $D_{1-\chi, k}$, and that the $k_{\chi}$ are continuous at $(\bar{x}, \bar{x})$. Then $\phi$ is not $\mathrm{C}^{1}$ at $(0, \bar{x})$.

Proof. Assume for contradiction that $\phi$ is $\mathrm{C}^{1}$ at $(0, \bar{x})$. Differentiate (4) wrt. $\kappa$ using the first order condition to find

$$
\frac{\partial \phi}{\partial \kappa}\left(\kappa, x_{\chi}, \chi\right)=\frac{\partial \phi}{\partial \kappa}\left(\kappa, \xi_{\chi}, 1-\chi\right)-\frac{\partial K_{\chi}}{\partial \kappa}\left(\kappa, x_{\chi}, \xi\right)-k_{\chi}\left(x_{\chi}, \xi_{\chi}\right)
$$

for $x_{\chi}$ in the sets $O$. Add ( 7$)$ for $\chi=0,1$ and let $\kappa \rightarrow 0$ to arrive at the contradiction

$$
\begin{aligned}
0 & =\lim \left(\frac{\partial \phi}{\partial \kappa}\left(\kappa, x_{0}, 0\right)+\frac{\partial \phi}{\partial \kappa}\left(\kappa, x_{1}, 1\right)-\frac{\partial \phi}{\partial \kappa}\left(\kappa, \xi_{0}, 1\right)-\frac{\partial \phi}{\partial \kappa}\left(\kappa, \xi_{1}, 0\right)\right) \\
& =k_{0}(\bar{x}, \bar{x})+k_{1}(\bar{x}, \bar{x})
\end{aligned}
$$

which is assumed non-zero by (3).

In other words, under the differentiability assumption and (1) - (6), then either the value function $\phi$ is non-robust, or the optimal strategy is.

We have chosen to give the theorem a very general formulation. The calculations give an instructive way of developing apparently stronger results in special cases. In particular, [2], [3], 
[5] and [6] all conclude that the $\kappa$-derivative is infinite at zero in their models, the latter two by methods similar to the approach to follow below. Rather than stating rigorous corollaries, we shall proceed to see how such improvements may be found. For example, in optimal switching problems where the state evolves as an Itô diffusion, we frequently know that the value function has the form

$$
\phi(\kappa, x, \chi)=\hat{A}_{\chi}(\kappa) \hat{\psi}_{\chi}(x)+\check{A}_{\chi}(\kappa) \check{\psi}_{\chi}(x)+\tilde{\psi}_{\chi}(x),
$$

solving the linear second order Hamilton-Jacobi-Bellman differential equations. Then

$$
\begin{aligned}
\lim ( & \left.\frac{\partial \phi}{\partial \kappa}\left(\kappa, x_{0}, 0\right)+\frac{\partial \phi}{\partial \kappa}\left(\kappa, x_{1}, 1\right)-\frac{\partial \phi}{\partial \kappa}\left(\kappa, \xi_{0}, 1\right)-\frac{\partial \phi}{\partial \kappa}\left(\kappa, \xi_{1}, 0\right)\right) \\
=\lim ( & \hat{A}_{0}^{\prime}(\kappa) \cdot\left(\hat{\psi}_{0}\left(x_{0}\right)-\hat{\psi}_{0}\left(\xi_{1}\right)\right)+\check{A}_{0}^{\prime}(\kappa) \cdot\left(\check{\psi}_{0}\left(x_{0}\right)-\check{\psi}_{0}\left(\xi_{1}\right)\right) \\
& \left.+\hat{A}_{1}^{\prime}(\kappa) \cdot\left(\hat{\psi}_{1}\left(x_{1}\right)-\hat{\psi}_{1}\left(\xi_{0}\right)\right)+\check{A}_{1}^{\prime}(\kappa) \cdot\left(\check{\psi}_{1}\left(x_{1}\right)-\check{\psi}_{1}\left(\xi_{0}\right)\right)\right) .
\end{aligned}
$$

For this limit to be nonzero, at least one of the $A^{\prime}$ must be unbounded near 0 .

Even more specifically, a wide class of one-dimensional pure regime switching problems studied in the literature, e.g. [7], has value function of the form (8) but with the $\check{A} \chi=0$ (which also is the typical case for problems without Brownian noise) and it is optimal to switch to regime 1 at $x=x^{*}$ and to regime 0 at $x=x_{*}<x^{*}$, where both trigger states tend to $\bar{x}$ at vanishing cost. Writing $\psi_{\chi}^{*}$ for $\hat{\psi}_{\chi}\left(x^{*}\right)$ and so forth, and $k^{*}:=k_{0}\left(x^{*}, x^{*}\right)$, and $k_{*}:=k_{1}\left(x_{*}, x_{*}\right)$, we immediately get

$$
\left(\begin{array}{c}
A_{0}^{\prime}(\kappa) \\
A_{1}^{\prime}(\kappa)
\end{array}\right)=\left(\psi_{0 *} \psi_{1}^{*}-\psi_{0}^{*} \psi_{1 *}\right)^{-1}\left(\begin{array}{c}
\psi_{1 *} k^{*}-\psi_{0}^{*} k_{*} \\
\psi_{0 *} k^{*}-\psi_{1}^{*} k_{*}
\end{array}\right)
$$

and in the limit

$$
A_{\chi}^{\prime}\left(0^{+}\right)= \pm\left(k_{0}(\bar{x}, \bar{x})+k_{1}(\bar{x}, \bar{x})\right) \cdot \lim _{\kappa \searrow 0}\left(\psi_{\chi^{*}}-\psi_{\chi}^{*}\right)^{-1}= \pm \infty
$$

if the derivative exists at all. In applications such as several of the aforementioned works, we may know from the problem that the value function is indeed monotone in $\kappa$, and then it is indeed valid to conclude that

$$
\frac{\partial \phi}{\partial \kappa}\left(0^{+}, x, \chi\right)= \pm \infty
$$

the sign of course depending on the parameterization.

Remark. A remark on the high contact principle is appropriate. The result presented herein does not directly make use of high contact. Nevertheless, there is a heuristic connection. In many impulse control, problems, (4) and high contact together determine the $A$ coefficients and the boundary simultaneously; therefore $\mathrm{C}^{1}$ regularity is connected to the phenomenon that the continuation region collapses to a single point in the sense of this paper. We also note that the high contact principle is extended to semimartingale processes, see [8]; while our setup is designed for Markov processes, one may allow the system to depend on a «memory parameter».

We end our discussion with another example pointing that the result herein may be applied in two stages: Consider the well-known Merton problem [9] of optimal portfolio choice in a frictionless market consisting of a safe bank account and a risky asset (stock) modeled by a 
geometric Brownian motion. The result is that the fraction invested in the risky asset should be kept constant, the so-called Merton line. Assuming power utility and introducing transaction costs proportional to each trade, $[10]$ shows that the optimal strategy is to keep the fraction invested in the risky assets between two fixed levels. By our results, the value function should be non-robust with respect to the transaction cost; indeed, in [11] it is shown that, in our notation sans regimes, $\phi\left(0^{+}, x\right)-\phi(\kappa, x)$ is of order $\kappa^{2 / 3}$ as $\kappa \searrow 0$. The case with both fixed and proportional transaction costs turns out to be harder and is considered in [12]; a numerical approximation suggests that the continuation region may converge to the case with only proportional transaction costs, in which case the value function, known to be continuous in the state variable, will be non-robust with respect to the fixed cost also.

\section{References}

[1] Menaldi, J-L.; Rofman, E. On stochastic control problems with impulse cost vanishing. In Lecture Notes in Econom. and Math. Systems; Fiacco, A.V., Kortanele, K.O., Eds.; Springer-Verlag: Berlin-New York, 1983. Vol. 215, 281-294.

[2] Øksendal, B. Stochastic Control Problems where Small Intervention Costs Have Big Effects, Appl. Math. Optim. 1999, 40 (3), 355-375.

[3] Øksendal, B.; Ubøe, J.; Zhang, T. Non-robustness of some impulse control problems with respect to intervention costs, Stochastic Anal. Appl. in press.

[4] Alvarez, L.H.R. Singular stochastic control, linear diffusions, and optimal stopping: A class of solvable problems, SIAM J. Control Optim. 2001, 39 (6), 1697-1710.

[5] Øksendal, A. A solvable irreversible investment problem with transaction costs, Preprint Pure Mathematics, Department of Mathematics, University of Oslo. 20001.

[6] Mundaca, G.; Øksendal, B. Optimal stochastic intervention control with application to the exchange rate, J. Math. Econom. 1998, 29 (2), 225-243.

[7] Brekke, K.A.; Øksendal, B. Optimal Switching in an Economic Activity Under Uncertainty, SIAM J. Control Optim. 199432 (4), 1021-1036.

[8] Chitashvili, R.J. On the smooth fit boundary conditions in the optimal stopping problem for semimartingales. In Lecture Notes in Control and Inform. Sci.; Christopeit, N., Helmes, K. and M. Kohlmann, Eds.; Springer-Verlag: Berlin, 1989. Vol 126, 82-90.

[9] Merton, R.C. Optimum consumption and portfolio rules in a continuous time model, J. Econom. Theory 19713 (4), 373-413.

[10] Davis, M.H.A.; Norman, A.R. Portfolio selection with transaction costs, Math. Oper. Res. $199015(4), 676-713$.

[11] Shreve, S.E.; Soner, H.M. Optimal investment and consumption with transaction costs, Ann. Appl. Probab. 19944 (3), 609-692.

[12] Øksendal, B.; Sulem, A.; Optimal consumption and portfolio with both fixed and proportional transaction costs, Preprint Pure Mathematics, Department of Mathematics, University of Oslo. 199919. 